Bundesgesundheitsbl $2021 \cdot 64: 378-383$ https://doi.org/10.1007/s00103-021-03289-x Eingegangen: 14 . September 2020

Angenommen: 26. Januar 2021

Online publiziert: 15. Februar 2021

(c) Der/die Autor(en) 2021

Katharina Wabnitz ${ }^{1,2}$. Sophia Galle ${ }^{3,5} \cdot$ Louise Hegge $e^{4,5} \cdot$ Oskar Masztalerz ${ }^{6}$. Eva-Maria Schwienhorst-Stich ${ }^{7} \cdot$ Michael Eichinger ${ }^{8,9,10}$

${ }^{1}$ Institut für Medizinische Informationsverarbeitung, Biometrie und Epidemiologie (IBE), Pettenkofer School of Public Health, Ludwig-Maximilians-Universität, München, Deutschland

${ }^{2}$ The Primary Care Unit, Department of Public Health and Primary Care, University of Cambridge, Cambridge, Großbritannien

${ }^{3}$ Julius-Maximilians-Universität Würzburg, Würzburg, Deutschland

${ }^{4}$ Technische Universität München, München, Deutschland

${ }^{5}$ Deutsche Allianz Klimawandel und Gesundheit (KLUG e.V.), Berlin, Deutschland

${ }^{6}$ Charité - Universitätsmedizin Berlin, Berlin, Deutschland

${ }^{7}$ Zentrum für Studiengangsmanagement und -entwicklung (ZSME), Julius-Maximilians-Universität Würzburg, Würzburg, Deutschland

${ }^{8}$ Mannheimer Institut für Public Health, Sozial- und Präventivmedizin, Medizinische Fakultät Mannheim der Universität Heidelberg, Mannheim, Deutschland

${ }^{9}$ Abteilung für Pädiatrische Epidemiologie, Institut für Medizinische Biometrie, Epidemiologie und Informatik, Universitätsmedizin der Johannes-Gutenberg-Universität Mainz, Mainz, Deutschland

${ }^{10} \mathrm{Klinik}$ für Kinder- und Jugendmedizin, Medizinische Fakultät Mannheim der Universität Heidelberg, Mannheim, Deutschland

\title{
Planetare Gesundheit - transformative Lehr- und Lernformate zur Klima- und Nachhaltigkeitskrise für Gesundheitsberufe
}

türlichen Habitaten - zum Teil durch Wildtierhandel verschärft - eng mit der menschlichen Gesundheit zusammenhängen kann [4]. Werden durch diese globalen Umweltveränderungen sogenannte planetare Belastungsgrenzen überschritten (- Infobox 1), steigt das Risiko, dass nicht-lineare Veränderungsprozesse und sich selbst verstärkende Feedbackmechanismen einsetzen, die durch menschliches Handeln nicht mehr aufzuhalten sind [5].

Während die Dringlichkeit der Klimaund Nachhaltigkeitskrise und ihre gesundheitlichen Auswirkungen im deutschen Gesundheitswesen bis vor Kurzem nur am Rande berücksichtigt wurden, rückt das Thema nun zunehmend in den Fokus. Dies zeigt sich nicht zuletzt an der Forderung des Deutschen Ärztetags 2019 nach einer Fokussierung auf die Klimakrise [6]. Auch auf gesundheitspoliti-
Infobox 1 Konzept der planetaren Belastungsgrenzen (Planetary Boundaries)

- Beschreibt Grenzwerte für die ökologische Tragfähigkeit 9 natürlicher Systeme oder Prozesse

- Grenzwerte beruhen auf wissenschaftlichen Erkenntnissen und dem Vorsorgeprinzip

- Kipppunkte (Tipping Points, empirisch belegt oder postuliert) markieren irreversible und rapide Veränderungen natürlicher Systeme und Prozesse mit potenziell katastrophalen Auswirkungen auf die Lebensbedingungen auf der Erde

- Beispiel für eine Belastungsgrenze in Bezug auf die Klimakrise: $\mathrm{CO}_{2}$-Konzentration in der Atmosphäre (aktuell ca. 410 Parts per Million [27], postulierter Kipppunkt $\geq 450$ Parts per Million [5]) führt wahrscheinlich zum Abschmelzen der Polkappen und Verlust von Permafrostböden, sodass sich die Erderwärmung exponentiell beschleunigen würde [28] 
scher Ebene wird der Klima- und Nachhaltigkeitskrise vermehrt Beachtung geschenkt, indem u.a. im Bundesministerium für Gesundheit ein Referat geschaffen wurde, das sich v. a. mit den gesundheitlichen Auswirkungen der Klima- und Nachhaltigkeitskrise beschäftigt [7].

\section{Lehre und Transformation}

Um den skizzierten Herausforderungen für die menschliche Gesundheit $\mathrm{zu}$ begegnen, muss die Aus-, Fort- und Weiterbildung von Fachkräften im Gesundheitswesen rasch und flächendeckend um Lehr- und Lernformate zur Klima- und Nachhaltigkeitskrise ergänzt werden [8]. Neben der Vorbereitung auf die Behandlung klimabedingter Gesundheitsprobleme, wie beispielsweise Hitzeschäden oder Dehydratation, sollten diese Lehr- und Lernformate insbesondere $\mathrm{zu}$ transformativem Handeln befähigen $[8,9]$.

Transformation meint die umfassende Veränderung aller gesellschaftlichen Strukturen und Sektoren, die aktuell die planetare Gesundheit bedrohen [10]. Dies schließt technische Lösungen wie die Elektromobilität oder die Energieerzeugung durch erneuerbare Energien, aber auch nachhaltige Verhaltensweisen bezüglich Konsum und Ernährung ein. Insbesondere braucht es zum Schutz der Gesundheit von Menschen heute und in der Zukunft eine Transformation unserer Werte und Haltungen, da diese wesentliche Bedingungsfaktoren für Veränderungen unseres Politik- und Wirtschaftssystems darstellen [11].

\section{Planetare Gesundheit}

Das Konzept der planetaren Gesundheit (planetary health) kann für die Gestaltung und Umsetzung gesellschaftlicher Transformationsprozesse Orientierung geben und dazu beitragen, dass die einzelnen Transformationsschritte am übergeordneten Ziel "gesunde Menschen auf einem gesunden Planeten“" ausgerichtet werden. Im Zentrum dieses Konzepts steht die wechselseitige Abhängigkeit zwischen dem Zustand der natürlichen Umwelt und der menschlichen Gesundheit [12]. Menschliche Gesundheit und Wohlergehen hängen unmittelbar mit globalen und lokalen Umweltveränderungen zusammen. Es geht also nicht allein um Umwelt- und Klimaschutz, sondern um den Erhalt der natürlichen Lebensgrundlagen und damit das Überleben der menschlichen Zivilisation.

Das Konzept greift außerdem zukunfts- und gerechtigkeitsbezogene Aspekte auf, indem es die Gesundheit zukünftiger Generationen als Zielgröße und gegenwärtige Ungleichheiten als Ursachen für Gesundheit und Krankheit berücksichtigt [13]. Planetare Gesundheit ist darüber hinaus eng mit den Zielen für nachhaltige Entwicklung (Sustainable Development Goals) der Vereinten Nationen verknüpft [14], die als eine Übersetzung des theoretischen Konzepts in konkrete, praktisch umsetzbare Ziele verstanden werden können. Die Prinzipien und Wertvorstellungen, die planetare Gesundheit auszeichnen, spiegeln sich in den grundsätzlichen Maximen der Ziele für nachhaltige Entwicklung wider, zu deren Umsetzung sich auch Deutschland mit der Neuauflage der Deutschen Nachhaltigkeitsstrategie 2017 sowie im Koalitionsvertrag 2018 verpflichtet hat $[15,16]$.

Wir skizzieren im Folgenden, inwiefern Angehörige der Gesundheitsberufe eine besondere Rolle für gesellschaftliche Transformation im Sinne der Ziele für nachhaltige Entwicklung spielen können und müssen. Wir diskutieren Kernherausforderungen für die Lehre zu planetarer Gesundheit und beschreiben ausgewählte Aspekte einer flächendeckenden Institutionalisierung einschlägiger Lehrund Lernformate in der Aus-, Fort- und Weiterbildung der Gesundheitsberufe.

\section{Angehörige der Gesundheits- berufe als Schlüsselfiguren transformativen Wandels}

Angehörige der Gesundheitsberufe wie Mediziner*innen, Pflegende oder Psycho-, Physio- und Ergotherapeut ${ }^{\star}$ innen sowie Fachkräfte im Öffentlichen Gesundheitsdienst haben das Potenzial, zu Schlüsselfiguren transformativen Wandels zu werden. Sie tragen eine besondere Verantwortung für das Wohlergehen ihrer Patient*innen sowie der gesamten
Bevölkerung und sind dem Grundsatz des Nichtschadens („primum non nocere") verpflichtet. Zudem findet sich in $\$ 1$ der Musterberufsordnung für Ärzt*innen in Deutschland die Verpflichtung zur Erhaltung der natürlichen Lebensgrundlagen [17]. Diese ethische Handlungsmaxime teilen Ärzt ${ }^{*}$ innen mit allen anderen Angehörigen der Gesundheitsberufe. In Zeiten der Klimaund Nachhaltigkeitskrise haben Fachkräfte des Gesundheitswesens durch dieses gemeinsame Ethos eine besondere Verantwortung, Transformationsbedarfe $\mathrm{zu}$ identifizieren und proaktiv Wandel anzustoßen.

Zudem sind Angehörige der Gesundheitsberufe durch das ihnen entgegengebrachte Vertrauen [18] in einer günstigen Position, auf unterschiedlichen Ebenen der Gesellschaft transformativen Wandel zu initiieren und aktiv mitzugestalten. Dies beginnt bei der Patient*innenversorgung durch Förderung nachhaltiger Verhaltensweisen mit positiven Gesundheitseffekten (co-benefits), wie zum Beispiel die Umstellung auf eine vorwiegend pflanzenbasierte Ernährung [19], und reicht bis zur Beratung zu gesundheits- und nachhaltigkeitsbezogenen Aspekten in politischen Prozessen.

Durch interdisziplinäre Zusammenarbeit und synergistisches Handeln können Angehörige der Gesundheitsberufe somit zur Transformation in allen gesellschaftlichen Sektoren beitragen.

\section{Zentrale Herausforderungen auf dem Weg zur planetaren Gesundheit}

Um ein weiteres Voranschreiten der Klima- und Nachhaltigkeitskrise zu verhindern, müssen 3 zentrale Herausforderungen (challenges) adressiert werden. Diese Herausforderungen betreffen, in Anlehnung an Whitmee et al. [12]:

1. die Vorstellungskraft in Bezug auf eine Vision von Gesundheit und Wohlergehen für alle Menschen innerhalb planetarer Belastungsgrenzen, also ein Leben im Einklang mit der Natur (imagination challenge),

2. das Wissen um die Zusammenhänge zwischen der Klima- und Nachhaltigkeitskrise und menschlicher 
Gesundheit (knowledge challenge) und

3. die Umsetzung der Maßnahmen, die für die Sicherstellung umfassender Gesundheit und Wohlergehen innerhalb planetarer Belastungsgrenzen notwendig sind (implementation challenge).

Die Herausforderung hinsichtlich der Vorstellungskraft bezieht sich auf die Fähigkeit, Visionen einer Gesellschaft $\mathrm{zu}$ entwickeln, in der ein gesundes und nachhaltiges Leben für alle Menschen innerhalb planetarer Belastungsgrenzen sichergestellt ist. Diese Visionen dürfen nicht ausschließlich auf zukünftigem technologischem Wandel fußen, sondern müssen in erster Linie fundamentale ökologische, soziale und ökonomische Determinanten menschlicher Gesundheit adressieren. Die Lehre zu planetarer Gesundheit sollte diese Herausforderung aufgreifen, indem sie Weltbilder und Wertvorstellungen, inklusive ihrer ethischen Implikationen, kritisch reflektiert und neu bewertet [20]. Angehörige der Gesundheitsberufe sollten bei der Entwicklung positiver Zukunftsvisionen begleitet werden sowie Konzepte und Kompetenzen erlernen, mit denen sie diese Visionen auch im beruflichen und privaten Umfeld anregen und begleiten können. Die Fähigkeit, sich eine Welt umfassender und dauerhafter Gesundheit für alle Menschen innerhalb planetarer Belastungsgrenzen und die dafür notwendigen Rahmenbedingungen vorzustellen, ist der erste Schritt zur Realisierung planetarer Gesundheit.

Die Herausforderung hinsichtlich des Wissens bezieht sich einerseits auf den Umstand, dass unser Verständnis der Zusammenhänge zwischen Umweltveränderungen und menschlicher Gesundheit zum Teil noch lückenhaft ist. Andererseits ist das vorhandene Wissen den gesellschaftlichen Entscheidungsträgerinnen häufig nur unzureichend zugänglich. Für die Lehre zur planetaren Gesundheit bedeutet dies, dass Angehörige der Gesundheitsberufe bei der Wissensaneignung und der kritischen Reflexion vorhandener Wissenslücken begleitet werden sollten. Sie müssen insbesondere in der Lage sein, die Auswir-

Bundesgesundheitsbl 2021 · 64:378-383 https://doi.org/10.1007/s00103-021-03289-x (c) Der/die Autor(en) 2021

K. Wabnitz · S. Galle · L. Hegge · O. Masztalerz · E.-M. Schwienhorst-Stich · M. Eichinger Planetare Gesundheit - transformative Lehr- und Lernformate zur Klima- und Nachhaltigkeitskrise für Gesundheitsberufe

\section{Zusammenfassung}

Die Dringlichkeit der Klima- und Nachhaltigkeitskrise und ihre Auswirkungen auf die menschliche Gesundheit rücken auch im deutschen Gesundheitswesen zunehmend in den Fokus. Um ein weiteres Fortschreiten der Klima- und Nachhaltigkeitskrise zu verhindern, sind tiefgreifende Transformationsprozesse in allen gesellschaftlichen Sektoren notwendig (z. B. Verkehr, Energieerzeugung, Ernährungssystem). Angehörige der Gesundheitsberufe haben auf Basis ihrer ethischen Verpflichtung des Nichtschadens und ihrer guten Vertrauenswerte in der Gesellschaft das Potenzial, einen wichtigen Beitrag zu den notwendigen Transformationsprozessen zu leisten. Damit sie dieses Potenzial zur Gänze ausschöpfen können, sollten Angehörige der Gesundheitsberufe im Rahmen ihrer Aus-, Fort- und Weiterbildung bei der Entwicklung von Kompetenzen begleitet werden, die zu transformativem Handeln befähigen. Wir führen in diesem Artikel in das Konzept der planetaren Gesundheit ein, das hierfür sowohl inhaltliche als auch ethische Orientierung gibt. Außerdem geben wir einen Überblick über aktuelle Lehr- und Lernformate und identifizieren Aspekte, die zur Weiterentwicklung der Lehre im Bereich planetare Gesundheit beitragen könnten.

Schlüsselwörter

Lehre · Gesundheitswesen · Klimawandel . Transformation $\cdot$ Nichtschaden

\section{Planetary health-transformative education regarding the climate and sustainability crises for health professionals}

\section{Abstract}

The urgency of the climate and sustainability crises and their health effects are receiving increasing attention in the German health system. To avoid further exacerbation of these crises, profound transformative processes in all sectors of society are needed (e.g. transport, energy production, and food systems). Based on the ethical imperative of non-maleficence and the high levels of trust in society, health professionals have great potential to make an important contribution to the necessary transformative processes. In order to fully harness this potential, health professionals should be supported in developing competencies to take transformative action during their pre- and postgraduate education and professional training. In this article, we introduce the concept of planetary health, as this concept provides orientation for this both ethically and with regards to the subject matter. Furthermore, we provide an overview of current teaching and learning formats and identify aspects that could contribute to further developing planetary health education.

\section{Keywords}

Education · Health system - Climate change . Transformation · Do no harm kungen globaler, regionaler und lokaler Umweltveränderungen auf die Gesundheit $\mathrm{zu}$ analysieren und hinsichtlich ihrer Tragweite einzuordnen. Zudem sollten sie Kompetenzen entwickeln, um dieses Wissen auch effektiv an Entscheidungsträger*innen kommunizieren $\mathrm{zu}$ können.

Die Herausforderung hinsichtlich der Umsetzung beschreibt die Schwierigkeit, vom Wissen ins Handeln zu kommen. Der Lückenschluss zwischen dem Wis- sen um die negativen Auswirkungen der Klima- und Nachhaltigkeitskrise und dem aktiven Anstoßen von Transformationsprozessen in Richtung planetarer Gesundheit ist eine Aufgabe, die auf individueller, institutioneller und gesamtgesellschaftlicher Ebene gelöst werden muss. Damit Fachkräfte im Gesundheitswesen aktiv dazu beitragen können, sollten ihre Rollen als Initiator*innen und Gestalter*innen von Transformationsprozessen (change agents) in einschlägi- 
Tab. 1 Beispiele für curriculare und extracurriculare Lehr- und Lernformate zu planetarer Gesundheit

\section{Wahlfächer zu planetarer Gesundheit}

Aufnahme von Inhalten zu planetarer Gesundheit in Pflichtcurricula

Vertiefungsangebote, die Studierenden und Auszubildenden eine individuelle Profilbildung im Bereich planetare Gesundheit ermöglichen (z. B. als Neigungsorientierung)

Transdisziplinäre Lehr- und Lernformate, die den Austausch zwischen Studierenden, Auszubildenden und Praxispartner*innen (z. B. Öffentlicher Gesundheitsdienst, Stadtplaner*innen) fördern und ggf. Praktika außerhalb des klinischen Versorgungssystems erlauben

Studiengangs- bzw. fakultätsübergreifende Angebote (z. B. Ringvorlesungen)

Förderung studentischer Initiativen (z. B. Arbeitsgruppen zu planetarer Gesundheit), ggf. mit Anrechnung der Teilnahme als Prüfungsleistung

gen Lehr- und Lernformaten reflektiert und gestärkt werden. Dazu ist ebenfalls die Entwicklung praktischer Kompetenzen wie effektiver Gesprächsführung notwendig.

Derzeit steht v. a. die Wissensvermittlung zu den Auswirkungen der Klimaund Nachhaltigkeitskrise im Fokus einschlägiger Lehr- und Lernformate [20]. Damit Angehörige der Gesundheitsberufe in der Aus-, Fort- und Weiterbildung in ihrer Rolle als aktive Gestalter*innen von Transformationsprozessen gestärkt werden, ist es jedoch notwendig, dass auch die Herausforderungen hinsichtlich der Vorstellungskraft und der Umsetzung verstärkt adressiert werden. Ziele der Lehre zu planetarer Gesundheit sollten damit gleichermaßen die Wissensaneignung, die Entwicklung praktischer Kompetenzen und die kritische Reflexion von Werten und Haltungen sein - jeweils unterstützt durch angepasste didaktische Konzepte [20]. Hierfür sind sowohl inhaltlich-didaktische als auch institutionelle Reformen notwendig, die transformatives und systembasiertes Lernen und Lehren zum Ziel haben [21].

\section{Lehre zu planetarer Gesundheit im Gesundheitswesen}

International findet die Lehre zu planetarer Gesundheit zunehmend Eingang in Aus-, Fort- und Weiterbildungscurricula. Die frei zugängliche Datenbank der Planetary Health Alliance, einem Konsortium aus internationalen Universitäten, Nichtregierungsorganisationen, Forschungsinstituten und Regierungsstellen, zählte im November 2020 etwa 120 einschlägige Lehr- und Lernangebote [22]. Neben der Umsetzung kon- kreter Lehr- und Lernangebote werden beispielsweise in Kanada aktuell kompetenzorientierte Lernziele zur planetaren Gesundheit für medizinische Curricula entwickelt [23].

Während planetare Gesundheit bisher kaum in der Aus-, Fort- und Weiterbildung des deutschen Gesundheitswesens abgebildet war, entstehen seit Kurzem auch hierzulande einschlägige Lehr- und Lernformate. Diese reichen von studentischen Initiativen über Wahlfächer (z. B. das Projekttutorium Planetary Health an der Humboldt-Universität zu Berlin, das Wahlfach Klimakrise und Gesundheit an der Medizinischen Fakultät Mannheim oder das Wahlfach Planetare Gesundheit an der Medizinischen Fakultät Würzburg) und Ringvorlesungen (z. B. die Ringvorlesung Umwelt an der Technischen Universität München oder die Ringvorlesung Planetary Health an der Ludwig-Maximilians-Universität München) bis zu standortübergreifenden Formaten wie die im Sommersemester 2020 virtuell abgehaltene Planetary Health Academy der Deutschen Allianz Klimawandel und Gesundheit (KLUG e. V.).

Der Erfolg dieser neu etablierten Angebote (z.B. über 3000 Anmeldungen für die Planetary Health Academy) zeigt, dass sie eine in Deutschland bis dato bestehende Lücke im Lehrangebot füllen. Nachdem die meisten Lehr- und Lernformate bisher im Umfeld medizinischer Fakultäten entstanden sind, sollten im nächsten Schritt weitere Angebote für alle Stufen der Ausbildung und alle weiteren Disziplinen des Gesundheitswesens, wie Zahnmedizin, Pflege, Therapiedisziplinen oder Public Health, etabliert werden. Dabei sind je nach fach- spezifischen Anforderungen und lokalen Gegebenheiten verschiedene Lehrund Lernformate vorstellbar, die in unterschiedlichem Ausmaß bei der Wissensaneignung, der Reflexion von Werten und Haltungen und bei der Entwicklung transformativer Kompetenzen unterstützen. Einige Beispiele für curriculare und extracurriculare Lehr- und Lernformate sind in $\bullet$ Tab. 1 zusammengefasst. - Tab. 2 gibt exemplarisch einen Überblick über das Wahlfach Klimakrise und Gesundheit, das seit September 2020 an der Medizinischen Fakultät Mannheim angeboten wird.

Parallel ist die Entwicklung von Weiter- und Fortbildungsformaten für Fachkräfte des Gesundheitswesens notwendig. Die Fortbildungspflicht für Fachärzt ${ }^{*}$ innen bietet beispielsweise eine gute Grundlage, um einen bedeutenden Anteil von Mediziner ${ }^{*}$ innen mit Fortbildungsangeboten zur planetaren Gesundheit zu erreichen.

Um die flächendeckende Etablierung einschlägiger Lehr- und Lernformate in der Aus-, Fort- und Weiterbildung zu unterstützen, sollten Entwicklungsprozesse an einzelnen Standorten durch standortübergreifende Institutionalisierungsprozesse begleitet werden.

Standortübergreifende Entwicklung und Verankerung verbindlicher Lernziele zur planetaren Gesundheit: Gemäß den oben beschriebenen Herausforderungen hinsichtlich der Vorstellungskraft, des Wissens und der Umsetzung sollten Lernziele zur planetaren Gesundheit neben der Wissensvermittlung insbesondere Kompetenzen abdecken, die für die Initiierung und Umsetzung wirksamer Transformationsprozesse notwendig sind. Dazu wäre die zeitnahe Verankerung von verbindlichen nationalen Lernzielen zur planetaren Gesundheit für alle Gesundheitsberufe sinnvoll. Die derzeit laufende Weiterentwicklung des Nationalen Kompetenzbasierten Lernzielkatalogs Medizin (NKLM) und des Gegenstandskatalogs Medizin (GK) bietet hierzu eine gute Gelegenheit.

Dabei könnten bereits etablierte Lehrprinzipien und ein international derzeit in Entwicklung befindlicher Musterlernzielkatalog als Ausgangspunkte dienen $[22,24]$. Auf Basis standortübergreifend 
Tab. 2 Überblick über den Inhalt und die Rahmenbedingungen des Wahlfachs Klimakrise und Gesundheit an der Medizinischen Fakultät Mannheim

\begin{tabular}{ll} 
Zeitraum & Inhalt \\
\hline Blockkurs (3 & Tage, jeweils in der letzten Woche der Semesterferien) \\
$\begin{array}{ll}\text { Tag 1, Vor- } \\
\text { mittag }\end{array}$ & Grundlagen zur Klimakrise \\
& Darstellung des Paris-kompatiblen deutschen \\
& $\mathrm{CO}_{2}$-Budgets und seiner Allokation über die Zeit \\
& Grundlegende Aspekte der Transformationsforschung \\
& (z.B. Hauptgutachten des Wissenschaftlichen Beirats \\
& Globale Umweltveränderungen, Transformationsarenen \\
nach Schneidewind) \\
Einführung in die planetare Gesundheit als umfassendes \\
Gesundheitskonzept
\end{tabular}

Tag 1, Nach- Einführung in die gesundheitlichen Auswirkungen der mittag Klimakrise

Ethische Dimensionen der Klimakrise und ihrer gesundheitlichen Auswirkungen

Tag 2, Vor- Einführung in praktische Aspekte transformativen Hanmittag delns

Beispiele zur praktischen Umsetzung von Transformationsprozessen in Kliniken und Kommunen

Tag 2, Nach- Einführung in studentische Praxisprojekte aus dem Bemittag reich Klima und Gesundheit als Prüfungsleistung Entwicklung erster Ideen zu Praxisprojekten

Tag 3, Vor- Vorstellung lokaler Initiativen aus dem Bereich Klimakrimittag se und Gesundheit (z. B. Health for Future)

Arbeit an den Praxisprojekten

Tag 3, Nach- Arbeit an den Praxisprojekten mittag

Täglich Eingangs- und Abschlussrunde

Termine während des Semesters (2 Termine, Dauer: jeweils $90 \mathrm{~min}$ )

Termin 1 Arbeit an den Praxisprojekten und Klärung offener Fragen

Termin 2 Präsentation der Praxisprojekte

\section{Prüfungsleistung}

Präsentation des Praxisprojekts als Kleingruppe im Rahmen des Wahlfachs

Schriftliche Reflexion zum Praxisprojekt gültiger Lernziele könnten anschließend an allen medizinischen Fakultäten vergleichbare Lehr- und Lernformate neu entwickelt bzw. bestehende Formate ergänzt werden. Ähnliche Prozesse sollten auch für andere Berufsgruppen im Gesundheitswesen angestoßen werden. Für die Zahnmedizin, Psychotherapie und Pharmazie könnte die Entwicklung bzw. Überarbeitung der spezifischen Gegenstandskataloge am Institut für medizinische und pharmazeutische Prüfungs-
Input durch Lehrende

Verkürztes Planspiel in

Kleingruppen

Input durch Lehrende

Input durch Gastdozierende und Reflexion in Kleingruppen sowie im Plenum

"Think-pair-share" und ergänzender Input durch Lehrende

Kleingruppenarbeit und Input durch Lehrende

Input durch Gastdozierende

Input durch Praxispartner*innen und anschließende Diskussion

Input durch Lehrende

Kleingruppenarbeit

Kurzinput durch Praxispartner*innen und Diskussion im Plenum

Kleingruppenarbeit

Kleingruppenarbeit

Kleingruppenarbeit und Diskussion im Plenum

Präsentation durch Kleingruppen und Reflexion im Plenum
Format/Methode

unterstützen. Durch einen modularen Aufbau der Mustercurricula könnte sichergestellt werden, dass diese nach Abschluss der Erprobungsphase verhältnismäßig einfach auf verschiedene Ausbildungseinrichtungen mit unterschiedlichen Studien- und Prüfungsordnungen übertragbar sind. Im Sinne des Constructive Alignment [25], d.h. der Ausrichtung nicht nur der Lehr- und Lernmethoden und -inhalte, sondern auch der Prüfungsformate an bestehenden Lernzielen, sollten außerdem Prüfungsformate entwickelt werden, die das Erreichen der übergeordneten Lernziele auf optimale Weise unterstützen. Aktuell erproben einzelne Standorte unterschiedliche Lehr- und Lernformate. Bei Interesse an diesen Entwicklungen steht die Korrespondenzautorin dieses Artikels sehr gerne zur Verfügung.

Kapazitätsaufbau unter Lehrenden: Lehre zu planetarer Gesundheit, wie in diesem Beitrag skizziert, geht weit über das gegenwärtige Lehrportfolio von Dozierenden in Bildungseinrichtungen des Gesundheitswesens hinaus [26]. Insbesondere die Begleitung von Studierenden, Auszubildenden und Fachkräften bei der Entwicklung von Kompetenzen, die für die Initiierung und Umsetzung von Transformationsprozessen notwendig sind, ist anspruchsvoll und setzt spezifische didaktische Fertigkeiten voraus. Vor diesem Hintergrund sollten Angebote geschaffen werden, die den Kapazitätsaufbau unter Dozierenden unterstützen (z.B. Etablierung (hochschul-)didaktischer Weiterbildungsangebote zum transformativen und erfahrungsbasierten Lernen). Dabei kann auch die inhaltliche und didaktische (Weiter-)Entwicklung von Lehr- und Lernformaten unter aktiver Einbindung von Studierenden und Auszubildenden unterstützend wirken [26].

Evaluation transformativer Lehrformate: Da transformative Lehrformate in den meisten Institutionen neu entwickelt werden müssen, sollte die Entwicklung im Rahmen von Lehrforschungsprojekten engmaschig evaluiert werden [20]. Ein standortübergreifendes Vorgehen könnte die Sichtbarkeit und Aussagekraft dieser Evaluationsergebnisse erhöhen. 


\section{Fazit}

Die Klima- und Nachhaltigkeitskrise bedroht die menschliche Gesundheit heute und in der Zukunft. Angehörige der Gesundheitsberufe haben eine ethische Verantwortung, Gesundheit zu schützen. Durch das in sie gesetzte Vertrauen besitzen sie zudem ein besonderes Potenzial, gesellschaftliche Transformation zu initiieren und zu gestalten. Damit Angehörige der Gesundheitsberufe jedoch als aktive Gestalter*innen des Wandels zu einem gesunden und nachhaltigen Leben für alle Menschen beitragen können, muss die Aus-, Fort- und Weiterbildung flächendeckend am Konzept der planetaren Gesundheit ausgerichtet werden. Die Zeit ist reif.

\section{Korrespondenzadresse}

\section{Katharina Wabnitz}

Institut für Medizinische Informationsverarbeitung, Biometrie und Epidemiologie (IBE), Pettenkofer School of Public Health, LudwigMaximilians-Universität

Elisabeth-Winterhalter-Straße 6, 81377 München, Deutschland

kathy.wabnitz@ibe.med.uni-muenchen.de

Danksagung. Wir danken Sabine Gabrysch, Martin Herrmann, Leonie Dudda und Laura-Marie Strützke für die kritische Durchsicht des Artikels und die zahlreichen wertvollen Kommentare.

Funding. Open Access funding enabled and organized by Projekt DEAL.

\section{Einhaltung ethischer Richtlinien}

Interessenkonflikt. K. Wabnitz, S. Galle, L. Hegge, O. Masztalerz, E.-M. Schwienhorst-Stich und M. Eichinger geben an, dass kein Interessenkonflikt besteht.

Für diesen Beitrag wurden von den Autoren keine Studien an Menschen oder Tieren durchgeführt. Für die aufgeführten Studien gelten die jeweils dort angegebenen ethischen Richtlinien.

Open Access. Dieser Artikel wird unter der Creative Commons Namensnennung 4.0 International Lizenz veröffentlicht, welche die Nutzung, Vervielfältigung, Bearbeitung, Verbreitung und Wiedergabe in jeglichem Medium und Format erlaubt, sofern Sie den/die ursprünglichen Autor(en) und die Quelle ordnungsgemäß nennen, einen Link zur Creative Commons Lizenz beifügen und angeben, ob Änderungen vorgenommen wurden.

Die in diesem Artikel enthaltenen Bilder und sonstiges Drittmaterial unterliegen ebenfalls der genannten Creative Commons Lizenz, sofern sich aus der Abbildungslegende nichts anderes ergibt. Sofern das be- treffende Material nicht unter der genannten Creative Commons Lizenz steht und die betreffende Handlung nicht nach gesetzlichen Vorschriften erlaubt ist, ist für die oben aufgeführten Weiterverwendungen des $\mathrm{Ma}$ terials die Einwilligung des jeweiligen Rechteinhabers einzuholen.

Weitere Details zur Lizenz entnehmen Sie bitte der Lizenzinformation auf http://creativecommons.org/ licenses/by/4.0/deed.de.

\section{Literatur}

1. Costello A et al (2009) Managing the health effects of climate change: Lancet and University College London Institute for Global Health Commission. Lancet 373(9676):1693-1733

2. Watts $N$ et al (2019) The 2019 report of The Lancet Countdown on health and climate change: ensuring that the health of a child born today is not defined by a changing climate. Lancet 394(10211):1836-1878

3. Nowak D (2019) Erderwärmung - ein Blick auf Deutschland. Dtsch Arztebl Int 116(31/32):519-520

4. Webster J, Borlase A, Rudge J (2017) Who acquires infection from whom and how? Disentangling multi-hostand multi-modetransmission dynamics in the 'elimination' era. Phil Trans R Soc B 372:20160091

5. Steffen W et al (2015) Sustainability. Planetary boundaries: guiding human development on a changing planet. Science 347(6223):1259855

6. Bundesärztekammer Deutschland (2019) 122. Deutscher Ärztetag Beschlussprotokoll. https:// www.bundesaerztekammer.de/fileadmin/user upload/downloads/pdf-Ordner/122.DAET/ 122DAETBeschlussprotokoll.pdf. Zugegriffen: 18 Nov. 2020

7. bee/aerzteblatt.de (2020) Spahn erweitert Ministerium um Fachabteilung für Klima und Gesundheitsschutz. https://www.aerzteblatt. de/nachrichten/108855/Spahn-erweitertMinisterium-um-Fachabteilung-fuer-Klima-undGesundheitsschutz. Zugegriffen: 14. Sept. 2020

8. Matthies-Wiesler Franziska et al (2019) Lancet countdown policy brief for Germany. https://www.bundesaerztekammer.de/ fileadmin/user_upload/downloads/pdf-Ordner/ Pressemitteilungen/20191114_Klimawandel/3_ Lancet_Countdown_Policy_brief_for_Germany German_v01b.pdf.Zugegriffen: 14.Sept. 2020

9. Maxwell J, Blashki G (2016) Teaching about climate change in medical education: an opportunity. JPublic Health Res 5(1):673-673

10. Wissenschaftlicher Beirat der Bundesregierung (2011) Welt im Wandel Gesellschaftsvertrag für eine Große Transformation. https://issuu. com/wbgu/docs/wbgu_jg2011?e=37591641/ 69400318. Zugegriffen: 18. Nov. 2020

11. Prescott $S$ et al (2018) The Canmore declaration: statement of principles for planetary health. Challenges 9(2):31

12. Whitmee $S$ et al (2015) Safeguarding human health in the Anthropocene epoch: report of The Rockefeller Foundation-Lancet Commission on planetary health. Lancet 386(10007):1973-2028

13. Foster A et al (2019) Planetary health ethics: beyond first principles. Challenges 10(1):14

14. United Nations General Assembly (2015) The 2030 agenda for sustainable development. https://sustainabledevelopment.un.org/content/ documents/21252030\%20Agenda\%20for
\%20Sustainable\%20Development\%20web.pdf. Zugegriffen: 18. Nov. 2020

15. Bundesregierung Deutschland (2017) Deutsche Nachhaltigkeitsstrategie (Aktualisierung 2018). https://www.bundesregierung.de/resource/ blob/975274/1546450/65089964ed4a2ab07c a8a4919e09e0af/2018-11-07-aktualisierungdns-2018-data.pdf?download=1. Zugegriffen: 25 Jan. 2021

16. Regierungskoalition CDU/CSU (2018) Ein neuer Aufbruch für Europa Eine neue Dynamik für Deutschland Ein neuer Zusammenhalt für unser Land. https://www. bundesregierung.de/resource/blob/975224/ 847984/5b8bc23590d4cb2892b31c987ad672b7/ 2018-03-14-koalitionsvertrag-data.pdf? download=1. Zugegriffen: 25. Jan. 2021 (Koalitionsvertrag vom 12. März 2018)

17. Bundesärztekammer Deutschland (2018) (Muster-)Berufsordnung für die in Deutschland tätigen Ärztinnen und Ärzte. https:// www.bundesaerztekammer.de/fileadmin/user upload/downloads/pdf-Ordner/MBO/MBO-AE. pdf.Zugegriffen: 18. Nov. 2020

18. Ipsos MORI (2019) Ipsos MORI Veracity Index. https://www.ipsos.com/ipsos-mori/en-uk/trustpoliticians-falls-sending-them-spiralling-backbottom-ipsos-mori-veracity-index. Zugegriffen: 15. Aug. 2020

19. Haines A et al (2009) Public health benefits of strategies to reduce greenhouse-gas emissions: overview and implications for policy makers. Lancet 374(9707):2104-2114

20. Schwerdtle PN et al (2020) 12 tips for teaching environmental sustainability to health professionals. Med Teach 42(2):150-155

21. Frenk J et al (2010) Health professionals for a new century: transforming education to strengthen health systems in an interdependent world. Lancet 376(9756):1923-1958

22. Planetary Health Alliance (2020) Planetary health education. https://planetaryhealthalliance.org/ education. Zugegriffen: 17. Nov. 2020

23. Health and Environment Adaptive Response Task force (HEART) (2020) Planetary health competencies. https://www.cfms.org/what-we-do/globalhealth/heart-competencies. Zugegriffen: 17 . Nov 2020

24. Stone SB, Myers SS, Golden CD (2018) Cross-cutting principles for planetary health education. Lancet Planet Health 2(5):e192-e193

25. Biggs J (1996) Enhancing teaching through constructive alignment. High Educ 32:347-364

26. Tun S, Wellbery C, Teherani A (2020) Faculty development and partnership with students to integrate sustainable healthcare into health professions education. Med Teach. https://doi.org/ 10.1080/0142159X.2020.1796950

27. Tans P et al (2020) Global CO2 levels. https://www. 2degreesinstitute.org. Zugegriffen: 17. Nov. 2020

28. Steffen W et al (2018) Trajectories of the earth system in the anthropocene. Proc Natl Acad Sci U SA 115(33):8252-8259 